\title{
Verletzungen auf dem Schulhof - Eine Analyse individueller und kontextueller Faktoren
}

\author{
Injuries in the Schoolyard: An Analysis of Individual and \\ Contextual Factors
}

(๑) $\circledast \Theta$

Autoren

Sven Stadtmüller, Andreas Klocke, Andrea Giersiefen, Robert Lipp

Institut

Forschungszentrum Demografischer Wandel (FZDW), Frankfurt University of Applied Sciences, Frankfurt am Main

\section{Schlüsselwörter}

Unfälle, Verletzungen, Schule, Schulhof, Kinder, Jugendliche

\section{Keywords}

accidents, injuries, school, schoolyard, children, youth

\section{Bibliografie}

DOI https://doi.org/10.1055/s-0043-112743

Online-Publikation: 31.8.2017

Gesundheitswesen 2018; 80: 183-190

(c) Georg Thieme Verlag KG Stuttgart · New York

ISSN 0941-3790

\section{Korrespondenzadresse}

Dr. Sven Stadtmüller

Frankfurt University of Applied Sciences

Forschungszentrum Demografischer Wandel (FZDW)

Nibelungenplatz 1

60318 Frankfurt am Main

sven.stadtmueller@fzdw.de

\section{ZUSAMMENFASSUNG}

Ziel der Studie Verletzungen bei Kindern und Jugendlichen zählen zu den dringendsten Public Health Problemen in Deutschland. Neben Unfällen im Haushalt und in der Freizeit ist insbesondere der Lebensbereich Schule von großer Bedeutung. Hinsichtlich der Einflussfaktoren auf Schulverletzungen bestehen jedoch erhebliche Forschungslücken. Hier setzt der Beitrag an und analysiert individuelle und kontextuelle Faktoren, die auf das Verletzungsgeschehen in der Schule Einfluss nehmen. Dabei werden speziell Verletzungen untersucht, die sich auf dem Schulhof ereignen.

Methodik Die Datengrundlage bildet die zweite Erhebungswelle der Panelstudie „Gesundheitsverhalten und Unfallgeschehen im Schulalter“ (GUS). Im Anschluss an eine Zufallsauswahl von allgemeinbildenden weiterführenden Schulen in 11 Bundesländern wurden 10089 Schüler/innen im Alter von 11-13 Jahren aus 138 Schulen im Klassenverbund mittels eines standardisierten elektronischen Fragebogens befragt. Neben dem
Unfall- und Verletzungsgeschehen deckt der Fragebogen eine Bandbreite anderer Themen, wie z. B. das Gesundheit- und Freizeitverhalten, ab und erhebt zugleich auch Informationen über die Schule. Die Einflussfaktoren auf Schulhofverletzungen werden anhand von multivariaten logistischen Mehrebenmodellen analysiert.

Ergebnisse In der zweiten Erhebungswelle berichteten 5,8\% der befragten Schüler/innen, sich in den letzten 12 Monaten mind. eine behandlungsbedürftige Verletzung auf dem Schulhof zugezogen zu haben. Zusammenhänge mit dem Verletzungsgeschehen auf dem Schulhof zeigen sich für individuelle Faktoren, wie für das Geschlecht (weiblich, $O R=0,53$ ), Mobbingerfahrungen $(O R=1,68)$, Schlafstörungen $(O R=1,07)$ und für die individuelle sportliche Aktivität $(O R=1,03)$. Obgleich die Varianz primär auf Individualmerkmale zurückgeht, spielen auch einzelne Kontextmerkmale eine Rolle: An Schulen, deren Zustand von den dort beschulten Schüler/innen besser eingeschätzt wird, ist auch das Verletzungsrisiko geringer $(\mathrm{OR}=0,60)$.

Schlussfolgerung Präventionsmaßnahmen lassen sich aufgrund dieser Ergebnisse gezielt sowohl auf Individual- als auch Kontextebene ausrichten: Zum einen sollte die Prävention von Mobbing vor diesem Hintergrund fokussiert, aber auch der Zustand der Schulen weiterhin in den Blick genommen werden.

\section{ABSTRACT}

Aims of the Study Injuries of children and adolescents rank among the most immediate problems in public health in Germany. Alongside accidents in the household and during leisure time, the school is of great importance. However, there are significant gaps in knowledge about the causes of school injuries. This is the starting point of this article, which analyses individual and contextual factors that influence the occurrence of injuries in schools and deals explicitly with injuries happening in the schoolyard.

Method The data foundation is the second wave of the panel study „Gesundheitsverhalten und Unfallgeschehen im Schulalter" (Health Behavior and Occurrence of Accidents at School Age) (GUS). Following a random sampling of secondary general education schools in 11 German states, 10089 pupils aged 11-13 years from 138 schools were surveyed within their respective classes via a standardized electronic questionnaire. 
Alongside accidents and injuries, the questionnaire covers a wide range of topics including health and recreational behavior and also surveys information about the schools. The factors influencing schoolyard accidents are analyzed using multivariate, logistic multilevel-models.

Results In the second wave, $5.8 \%$ of the pupils reported at least one injury happening in the schoolyard in the last 12 months that had to be treated by a doctor. Correlations with these schoolyard accidents were found for individual factors such as gender (female, $O R=0.53$ ), experiences of bullying $(O R=1.68)$, sleeping problems $(\mathrm{OR}=1.07)$ and individual athletic activity $(O R=1.03)$. Although the variance is primarily tied to the individual level, certain contextual variables also proved influential: Schools, in which the overall condition is rated better by the pupils, also have a lower overall risk of injuries $(O R=0.60)$.

Conclusions According to these results, preventive measures may target the individual as well as the contextual level: prevention of bullying should be a focus in the light of these findings, but at the same time, also the condition of the schools should be taken into consideration.

\section{Einleitung}

Unfälle im Kindes- und Jugendalter gehören zu den dringendsten Public Health-Problemen in Deutschland. Sie zählen zu den häufigsten Todesursachen und rund 280000 Kinder und Jugendliche müssen pro Jahr aufgrund von Unfällen und Verletzungen stationär behandelt werden [1]. Neben Unfällen im Haushalt und in der Freizeit, ist insbesondere der Lebensbereich Schule von großer Bedeutung. So weist die Statistik der Deutschen Gesetzlichen Unfallversicherung (DGUV) für das Jahr 2014 bundesweit knapp 1,4 Millionen ärztlich versorgter Schulunfälle aus [2].

Bislang ist nur wenig darüber bekannt, welche Merkmale das Risiko beeinflussen, Unfälle und Verletzungen im Schulkontext zu erleiden. Dies liegt einerseits daran, dass die Mehrzahl der Untersuchungen zu Schulunfällen und -verletzungen auf Informationen basiert, die nur von den tatsächlich verletzten Schulkindern vorliegen bzw. erhoben wurden. Andererseits sind die wenigen nationalen wie internationalen epidemiologischen Studien, in deren Rahmen Kinder mit und ohne Verletzungen untersucht werden, eher darauf ausgelegt, Risikoprofile von Verletzungen im Allgemeinen zu skizzieren [3,4].

Im Rahmen der Studie Gesundheitsverhalten und Unfallgeschehen im Schulalter (GUS; unterstützt von der Deutschen Gesetzlichen Unfallversicherung (DGUV) ) werden ganz gezielt Verletzungen in der Schule in den Fokus gerückt und deren Einflussgrößen untersucht. Die Studie ist zudem als Panel angelegt, um kausale Strukturen von Schulverletzungen besser aufdecken zu können als es mit Querschnittstudien oder Trenddesigns möglich ist.

Die GUS-Studie unterscheidet in der Gruppe der Schulverletzungen nach Verletzungen, die sich auf dem Schulweg, im Schulsport, im Schulgebäude und auf dem Schulhof zugetragen haben. All diese Verletzungen weisen je eigene Risikokonstellationen aus individuellen und kontextuellen Faktoren auf. Wir konzentrieren uns im Folgenden auf Verletzungen auf dem Schulhof. Neben Verletzungen im Schulsport ist der Schulhof jener Ort im Kontext Schule, an dem sich die meisten Verletzungen zutragen [5-9]. Daher möchten wir im vorliegenden Beitrag auf der Grundlage multivariater Mehrebenenmodelle potenzielle Einflussfaktoren auf Verletzungen auf dem Schulhof untersuchen.

\section{Methodik}

Die GUS-Studie startete mit der ersten Erhebung im Schuljahr 2014/15 mit der Befragung von Schüler/innen der 5. Jahrgangsstu- fe. Im Rahmen der zweiten Erhebungswelle, auf deren Daten im Folgenden zurückgegriffen wird, konnten 10089 Schüler/innen der 6. Jahrgangsstufe aus 547 Schulklassen und 138 Schulen befragt werden.

\section{Stichprobendesign}

Alle Schüler/innen die im Schuljahr 2014/15 an allgemeinbildenden, weiterführenden Regelschulen in der 5 . Jahrgangsstufe unterrichtet wurden, stellen die Grundgesamtheit dar. Da von diesen Schulkindern keine Liste zur Stichprobenziehung vorliegt, erfolgte eine Auswahl von Schulen. Innerhalb der gezogenen Schulen wurde aus forschungspragmatischen Gründen die gesamte Jahrgangsstufe befragt.

Um sowohl alle Bundesländer zu berücksichtigen als auch die bundeslandspezifische Verteilung der Schüler/innen auf die einzelnen Schulformen adäquat abzubilden, wurde in enger Kooperation mit GESIS - Leibniz-Institut für Sozialwissenschaften eine geschichtete Zufallsstichprobe gezogen. Die Schichten repräsentieren dabei jeweils eine Kombination aus den Merkmalen Bundesland, Kreis, Schulform, Schulgröße und Urbanität. Die Bruttostichprobe für die erste Erhebungswelle betrug 854 Schulen in den 11 teilnehmenden Bundesländern. ${ }^{1}$ Knapp ein Fünftel der kontaktierten Schulen (17,3\%) konnte für die Teilnahme gewonnen werden. ${ }^{2}$

\section{Datenerhebung und Fragebogeninhalte}

Die Schüler/innen wurden innerhalb von 45 Minuten mittels eines standardisierten elektronischen (Offline-) Fragebogens im Klassenverbund befragt. Dabei erhielt jedes Schulkind, für das eine schriftliche Einwilligungserklärung der Eltern bzw. Erziehungsberechtigten vorlag, einen Tablet-PC ausgehändigt, auf dem der Fragebogen bereits aufgespielt war und selbständig beantwortet werden konnte. ${ }^{3}$ Bei sämtlichen Klassenbefragungen war ein(e) geschulte(r) Intervie-

\footnotetext{
${ }^{1}$ Mit Ausnahme von Hamburg und Bayern wurde die Studie in allen Bundesländern genehmigt. Aufgrund der 6-jährigen Grundschulzeit wurde in Brandenburg, Berlin und Mecklenburg-Vorpommern auf eine Befragung in den ersten beiden Wellen verzichtet. Diese Bundesländer steigen erst zur dritten Erhebungswelle (Befragung der 7. Jahrgangsstufe im Schuljahr 2016/17) in das Projekt ein.

2 Hierbei zeigt sich eine regional weitgehend gleichmäßige Verteilung des Rücklaufs. Hinsichtlich der Schulformen ist jedoch eine höhere Responserate bei Gymnasien zu beobachten.

3 Dabei wurde der Fragebogen unter Verwendung des Datenerhebungstools harvestyourdata programmiert und anschließend über eine zur Software zugehörige Applikation auf die Tablets gespielt. Dort konnte er dann offline von den Kindern beantwortet werden.
} 
wer * in anwesend, um die Kinder in die Befragung einzuführen, das Handling der Geräte zu erläutern und auf Fragen zu reagieren.

Im ersten Teil des Fragebogens wurden die Kinder nach erlittenen Verletzungen im Schulkontext befragt. Anschließend wurden das Bewegungs- und Ernährungsverhalten der Kinder erhoben, ebenso wie ihr physischer Gesundheitszustand oder Beeinträchtigungen, wie z. B. Schlaf- oder Konzentrationsstörungen. Neben soziodemografischen Angaben (z. B. Migration, materielle Ausstattung des Haushalts) wurden zudem Informationen über den Kontext Schule gewonnen. Hierzu zählen bspw. Angaben zum wahrgenommenen Zustand der Schule. Schließlich wurde der Datensatz mit weiteren Strukturmerkmalen der teilnehmenden Schulen angereichert (z. B. Schulform und Bundesland).

\section{Analysestrategie}

Die abhängige Variable stellen Verletzungen dar, die sich auf dem Schulhof ereignet haben. Die Analyse beschränkt sich auf die zweite Erhebungswelle, da der Berichtszeitraum von Schulverletzungen die letzten 12 Monate umfasst. Dies hat zur Folge, dass sich viele der in der ersten Welle von den Kindern berichteten Schulverletzungen noch während der Grundschulzeit ereignet haben dürften. In der zweiten Erhebungswelle ist hingegen gesichert, dass sich die Angaben zu erlittenen Schulverletzungen auf Verletzungen an der weiterführenden Schule beziehen, zu der Kontextmerkmale erhoben wurden.

Zur Erklärung von Verletzungen auf dem Schulhof werden einige Individual- und Kontextmerkmale herangezogen, deren Messung, Konstruktion und Verteilung in $>$ Tab. 1 dargestellt ist. ${ }^{4}$ Da die vorliegenden Daten eine hierarchische Struktur aufweisen, ist die Schätzung von Mehrebenenmodellen [10,11] notwendig. Diese Modelle tragen dem Umstand Rechnung, dass sich Schüler/innen derselben Schule ähnlicher sein sollten, als Schüler/innen aus unterschiedlichen Schulen.

Prinzipiell können in den Daten der GUS-Studie 3 Ebenen unterschieden werden: Die Schüler/innen (Ebene 1) sind eingebettet in Schulklassen (Ebene 2), die wiederum im Kontext Schule (Ebene 3) aufgehen. Bei näherer Betrachtung der Daten im Rahmen sogenannter Varianz-Komponenten-Modelle zeigt sich jedoch, dass es zur Analyse des Verletzungsgeschehens auf dem Schulhof ausreicht, nur die Schulen als Kontexte zu berücksichtigen. Bei anderen Verletzungen im Schulkontext, bspw. bei Verletzungen im Schulsport, ist jedoch die Berücksichtigung des Klassenkontexts geboten, während auf den übergeordneten Kontext Schule verzichtet werden kann.

\section{Ergebnisse}

Das erste Modell in $>$ Tab. 2 entspricht dem bereits angesprochenen Varianz-Komponenten-Modell, d. h. einem Mehrebenenmodell ohne Kovariaten, das lediglich Auskunft über die Konstante und deren Variation auf Kontextebene gibt.

Die darin ausgewiesene Varianz der Konstanten zeigt an, wie stark die Verletzungsquoten auf dem Schulhof zwischen den teilnehmenden Schulen streuen und kann dazu genutzt werden, den

${ }^{4}$ Da Fälle mit mind. einem fehlenden Wert auf den interessierenden Variablen aus der Analyse ausgeklammert wurden, reduziert sich die berücksichtigte Fallzahl für die geschätzten Modelle auf 7413 Schüler/innen.
Varianzanteil von Verletzungen auf dem Schulhof, der auf die Ebene der Schule entfällt, zu berechnen. Der sogenannte Variance-Partition-Koeffizient (VPC) beläuft sich auf 5,3\% (95\%-Konfidenzintervall: $2,6-10,7 \%) .{ }^{5}$ Somit sind Unterschiede in der Verletzungshäufigkeit von Schüler/innen auf dem Schulhof zwar in weiten Teilen auf Individualmerkmale zurückzuführen, jedoch spielt auch der Kontext Schule eine Rolle.

In Modell 2 sind nun zunächst ausschließlich Individualmerkmale als Prädiktoren des Verletzungsgeschehens auf dem Schulhof integriert. Dabei wurden diskrete Merkmale zentriert, um eine sinnvolle Interpretation der Konstanten zu ermöglichen. Die Effektstärken sind zusätzlich anhand der Odds-Ratios ausgewiesen. Können diese bei dichotomen Merkmalen direkt miteinander verglichen werden, so ist dies bei diskreten Merkmalen nicht möglich. ${ }^{6}$ Da in - Tab. 1 jedoch sowohl die Minima und Maxima als auch die Werte des 25 . und 75. Perzentils ausgewiesen sind, können diese dazu genutzt werden, vergleichbare Odds-Ratios zu kalkulieren.

Positive Effekte auf die logarithmierten Odds von Verletzungen auf dem Schulhof gehen von der Expositionszeit in der Schule, der sportlichen Aktivität in Verein und Freizeit sowie von der Prävalenz von Schlafstörungen aus. ${ }^{7}$ Kinder, die in den vergangenen 12 Monaten häufig gemobbt wurden, weisen ebenfalls eine höhere logarithmierte Chance auf, sich (mind.) eine Verletzung auf dem Schulhof zugezogen zu haben, als Kinder ohne Mobbingerfahrungen. Während sich weiterhin Jungen weitaus häufiger auf dem Schulhof verletzen als Mädchen, gehen von der materiellen Ausstattung des elterlichen Haushalts und vom Migrationshintergrund keine Effekte auf das Verletzungsgeschehen auf dem Schulhof aus. Gleiches gilt auch für das individuelle Risikoverhalten, dessen Einfluss allerdings die Grenze zu einer Vertrauenswahrscheinlichkeit von $95 \%$ nur knapp verfehlt.

Schließlich wird aus Modell 2 ersichtlich, dass sich die Varianz auf Kontextebene, vermutlich aufgrund von Kompositionseffekten, zwar verringert hat, das Gros der Varianz jedoch bestehen bleibt. Daher werden im dritten Modell nun zusätzlich Merkmale der Schule als Prädiktoren integriert.

Hier wird zunächst deutlich, dass weder die Region noch die Schulform einen signifikanten Einfluss auf die Zielvariable ausüben. Auch vom Anteil an Kindern mit Migrationshintergrund, vom Jungenanteil und vom Anteil an Schulkindern, die häufig gemobbt werden, gehen keine zusätzlichen, d. h. über die Individual- und Kompositionseffekte dieser Merkmale hinausgehenden Einflüsse auf Verletzungen auf dem Schulhof aus.

\footnotetext{
5 Hierzu wird die Varianz der Konstanten an der Gesamtvarianz relativiert. In logistischen Regressionsmodellen wird die Varianz auf Individualebene auf $\pi^{2} / 3$ fixiert, sodass der VPC auf der Grundlage dieses Modells mit 0,19/ $(3,29+0,19)$ berechnet wird [10].

${ }^{6}$ Bei kategorialen Merkmalen wurde stets die Modalkategorie als Referenzkategorie festgelegt.

7 Den Autoren ist bewusst, dass Merkmale, die auf wöchentlicher Basis erfasst wurden, nur schlecht mit der abhängigen Variablen (die einen Berichtszeitraum von 12 Monaten aufweist) in Beziehung zu setzen sind. Für die Messung von Merkmalen wie Schlaf- oder Konzentrationsstörungen wurde jedoch auf einen längeren Recallzeitraum verzichtet, um die Kinder nicht zu überfordern. Zudem besteht die Hoffnung, dass die Angaben der Kinder für die letzte Woche die vergangenen 12 Monate in gewisser Weise repräsentieren.
} 
- Tab. 1 Übersicht der berücksichtigten Merkmale und deren Verteilung.

\begin{tabular}{|c|c|c|c|c|c|c|c|}
\hline \multicolumn{8}{|l|}{ Abhängige Variable } \\
\hline Merkmal & Konstruktion & $\bar{x} / p$ & sd & $\min$ & $\max$ & $p(25)$ & $p(75)$ \\
\hline $\begin{array}{l}\text { Verletzung auf dem } \\
\text { Schulhof }\end{array}$ & $\begin{array}{l}\text { F: Wie häufig hast Du Dich seit der letzten Befragung (etwa } \\
\text { vor } 12 \text { Monaten) in der Schule oder auf dem Schulweg } \\
\text { verletzt und musstest deswegen von einem Arzt untersucht } \\
\text { bzw. behandelt werden? A: Ich habe mich während der letzten } \\
12 \text { Monate in der Schule nicht verletzt; 1-mal; 2-mal; 3-mal oder } \\
\text { häufiger } \\
\text { F: Wo genau warst Du gerade, als Dir diese Verletzung } \\
\text { passiert ist? A: im Sportunterricht; auf dem Schulweg (Hin- oder } \\
\text { Rückweg); auf dem Schulhof; im Schulgebäude, aber nicht im } \\
\text { Sportunterricht (z. B. im Klassenzimmer oder im Treppenhaus); an } \\
\text { der Bus- oder Bahnhaltestelle; bei einer Schulveranstaltung (z. B. } \\
\text { Schulausflug oder Projekttag usw.); an einem anderen Ort, und } \\
\text { zwar: } \\
\text { Zusätzlich wurde noch eine weitere Kontrollfrage nach der } \\
\text { ärztlichen Behandlung gestellt. Bei Kindern mit mehreren } \\
\text { Schulverletzungen wurden nur Informationen zur schwersten } \\
\text { Schulverletzung erhoben. Die Kinder wurden dabei gebeten, } \\
\text { an jene Verletzung zu denken, bei der die Genesung am längs- } \\
\text { ten gedauert hat. } \\
\text { Kinder, deren (schwerste) Schulverletzung sich laut } \\
\text { Selbstauskunft auf dem Schulhof zugetragen hat, erhielten } \\
\text { den Wert 1. }\end{array}$ & $5,8 \%$ & 0,2 & 0 & 1 & 0 & 0 \\
\hline \multicolumn{8}{|c|}{ Unabhängige Variablen (Individualmerkmale; $\mathbf{N}=7.413$ ) } \\
\hline Merkmal & Konstruktion & $\bar{x} / p$ & sd & $\min$ & $\max$ & $p(25)$ & $p(75)$ \\
\hline $\begin{array}{l}\text { Expositionszeit in der } \\
\text { Schule }\end{array}$ & $\begin{array}{l}\text { F: Von wann bis wann bist Du an einem Montag in der Schule? } \\
\text { Denke bitte auch an Hausaufgabenbetreuung, AGs usw. Trage } \\
\text { bitte die Uhrzeit ein. Wenn sich die Zeiten manchmal ändern, } \\
\text { denke bitte an die aktuelle Woche. } \\
\text { F: Denke jetzt bitte an den Dienstag: Um wie viel Uhr kommst Du } \\
\text { an der Schule an und um wie viel Uhr verlässt Du da die Schule? } \\
\text { (entsprechend auch für die übrigen Wochentage) } \\
\text { Die täglichen Expositionszeiten wurden aufsummiert und in } \\
\text { eine neue Variable überführt. }\end{array}$ & 30,6 & 3,4 & 19 & 47,3 & 28,2 & 32,6 \\
\hline $\begin{array}{l}\text { Sport in Verein und } \\
\text { Freizeit }\end{array}$ & $\begin{array}{l}\text { F: Wie viele Stunden Sport treibst Du in einer normalen Woche in } \\
\text { einem Verein? Wenn Du nicht in einem Sportverein bist, trage } \\
\text { einfach eine „O“ ein. Bitte runde auf volle Stunden auf! } \\
\text { F: Und wie viele Stunden Sport treibst Du in einer normalen } \\
\text { Woche in Deiner sonstigen Freizeit? Runde bitte wieder auf volle } \\
\text { Stunden auf! } \\
\text { Die Sportstunden in Verein und Freizeit wurden aufsummiert } \\
\text { und in eine neue Variable überführt. }\end{array}$ & 6,8 & 4,8 & 0 & 36 & 3 & 9 \\
\hline Schlafstörungen & $\begin{array}{l}\text { F: Wenn Du an die letzte Woche denkst, wie häufig treffen da } \\
\text { die folgenden Dinge auf Dich zu? Ich konnte schlecht } \\
\text { schlafen. A: gar nicht; an einem Tag; an 2-4 Tagen; an 5-6 } \\
\text { Tagen; jeden Tag } \\
\text { Die einzelnen Werte wurden in Wochentage überführt (gar } \\
\text { nicht=0; an einem Tag = 1; an 2-4 Tagen = 3; an 5-6 } \\
\text { Tagen = 5,5; jeden Tag=7) }\end{array}$ & 1,5 & 2,1 & 0 & 7 & 0 & 3 \\
\hline Risikobereitschaft & $\begin{array}{l}\text { F: Nun möchten wir gerne wissen, wie sehr die folgenden } \\
\text { Aussagen auf Dich zutreffen. (1) Ich habe Spaß daran, } \\
\text { gefährliche Sachen zu machen. (2) Ich liebe neue und } \\
\text { aufregende Erlebnisse, auch wenn sie manchmal etwas } \\
\text { gefährlich oder bedrohlich sind. A: trifft überhaupt nicht zu; } \\
\text { trifft eher nicht zu; teils/teils; trifft eher zu; trifft voll und ganz zu } \\
\text { Die Antwortoptionen wurden in einen Wertebereich von } 0 \\
\text { (trifft überhaupt nicht zu) bis } 4 \text { (trifft voll und ganz zu) } \\
\text { überführt und die Summe aus beiden Antworten gebildet. }\end{array}$ & 3,4 & 2,2 & 0 & 8 & 2 & 5 \\
\hline
\end{tabular}


> Tab. 1 Fortzetzung

\begin{tabular}{|c|c|c|c|c|c|c|c|}
\hline \multicolumn{8}{|c|}{ Unabhängige Variablen (Individualmerkmale, Fortsetzung) } \\
\hline Merkmal & Konstruktion & $\bar{x} / p$ & sd & $\min$ & $\max$ & $p(25)$ & $p(75)$ \\
\hline \multirow{5}{*}{ Mobbing } & $\begin{array}{l}\text { (nach einer ausführlichen Erläuterung des Begriffs Mobbing) F: } \\
\text { Wie oft bist Du in den letzten } 12 \text { Monaten von Mitschülerin- } \\
\text { nen und Mitschülern gemobbt worden? A: gar nicht; 1- oder } \\
\text { 2-mal im Jahr; 2- bis 3-mal im Monat; ungefähr 1-mal pro } \\
\text { Woche; mehrmals pro Woche. }\end{array}$ & & & & & & \\
\hline & $\begin{array}{l}\text { Aus den Antwortoptionen wurden } 3 \text { dichotome Variablen } \\
\text { gebildet: }\end{array}$ & & & & & & \\
\hline & $\begin{array}{l}\text { (1) Das Schulkind gab an, in den vergangenen } 12 \text { Monaten } \\
\text { nicht gemobbt worden zu sein }\end{array}$ & $70,7 \%$ & 0,5 & 0 & 1 & 0 & 1 \\
\hline & $\begin{array}{l}\text { (2) Das Schulkind gab an, 1- oder 2-mal im Jahr gemobbt } \\
\text { worden zu sein }\end{array}$ & $17,5 \%$ & 0,4 & 0 & 1 & 0 & 0 \\
\hline & $\begin{array}{l}\text { (3) Das Schulkind gab an, 2- bis 3-mal im Monat oder häufiger } \\
\text { gemobbt worden zu sein }\end{array}$ & $11,7 \%$ & 0,3 & 0 & 1 & 0 & 0 \\
\hline $\begin{array}{l}\text { (männliches) } \\
\text { Geschlecht }\end{array}$ & $\begin{array}{l}\text { F: Bist Du ein Junge oder ein Mädchen? A: Junge; Mädchen } \\
\text { Mädchen wurden mit dem Wert } 0 \text { kodiert, Jungen mit dem } \\
\text { Wert } 1\end{array}$ & $46,8 \%$ & 0,5 & 0 & 1 & 0 & 1 \\
\hline Migrationshintergrund & $\begin{array}{l}\text { F: In welchem Land wurde Deine Mutter geboren? F: In } \\
\text { welchem Land wurde Dein Vater geboren? A: (jeweils) in } \\
\text { Deutschland; in einem anderen Land, und zwar } \\
\text { Aus den Angaben der Schulkinder wurde eine dichotome } \\
\text { Variable gebildet, die dann den Wert } 1 \text { annimmt, wenn mind. } \\
\text { ein Elternteil nicht in Deutschland geboren wurde. }\end{array}$ & $28,4 \%$ & 0,5 & 0 & 1 & 0 & 1 \\
\hline $\begin{array}{l}\text { materielle Ausstattung } \\
\text { des elterlichen } \\
\text { Haushalts }\end{array}$ & $\begin{array}{l}\text { F: Besitzt Deine Familie ein Auto? A: nein; ja, eins; ja, } 2 \text { oder } \\
\text { mehr F: Hast Du ein eigenes Zimmer nur für Dich allein? A: } \\
\text { nein; ja F: Wie häufig bist Du mit Deiner Familie im letzten } \\
\text { Jahr in den Urlaub gefahren? A: überhaupt nicht; 1-mal; } \\
\text { 2-mal; mehr als 2-mal F: Wie viele Bücher gibt es ungefähr bei } \\
\text { Dir zu Hause? Zähle bitte keine Zeitschriften, Zeitungen oder } \\
\text { Schulbücher mit! A: keine oder sehr wenige (0-10 Bücher); etwa } \\
\text { ein Bücherbrett (11-25 Bücher); etwa ein Regal (26-100 Bücher); } \\
\text { etwa } 2 \text { Regale (101-200 Bücher); } 3 \text { oder mehr Regale (mehr als } \\
200 \text { Bücher) } \\
\text { Die Antworten wurden jeweils mit Punktwerten von } 0 \text { bis } \\
\text { maximal } 4 \text { versehen. Im Anschluss wurden alle Punktwerte } \\
\text { z-standardisiert und die Summe der z-Werte gebildet. }\end{array}$ & 0,0 & 2,4 & $-10,6$ & 3,2 & $-1,4$ & 1,5 \\
\hline \multicolumn{8}{|c|}{ Unabhängige Variablen (Kontextmerkmale; $\mathrm{N}=138$ ) } \\
\hline \multirow{4}{*}{ Region } & $\begin{array}{l}\text { Die Region, in der sich die Schule befindet, wird anhand von } 3 \\
\text { dichotomen Variablen erfasst. }\end{array}$ & & & & & & \\
\hline & $\begin{array}{l}\text { Norden (Schulen aus Schleswig-Holstein, Niedersachsen, } \\
\text { Bremen, NRW) }\end{array}$ & $39,1 \%$ & 0,5 & 0 & 1 & 0 & 1 \\
\hline & $\begin{array}{l}\text { Süden (Schulen aus Rheinland-Pfalz, Hessen, Saarland, } \\
\text { Baden-Württemberg) }\end{array}$ & $50,0 \%$ & 0,5 & 0 & 1 & 0 & 1 \\
\hline & Osten (Schulen aus Thüringen, Sachsen, Sachsen-Anhalt) & $10,9 \%$ & 0,3 & 0 & 1 & 0 & 0 \\
\hline \multirow{4}{*}{ Schulform } & $\begin{array}{l}\text { Auch für die Schulform wurden } 3 \text { dichotome Variablen } \\
\text { gebildet. }\end{array}$ & & & & & & \\
\hline & Gymnasien & $45,7 \%$ & 0,5 & 0 & 1 & 0 & 1 \\
\hline & $\begin{array}{l}\text { Gesamtschulen (beinhaltet sowohl kooperative als auch } \\
\text { integrierte Gesamtschulen) }\end{array}$ & $24,6 \%$ & 0,4 & 0 & 1 & 0 & 1 \\
\hline & $\begin{array}{l}\text { Sonstige Schulformen (z. B. Haupt- und Realschulen, } \\
\text { Oberschulen) }\end{array}$ & $29,7 \%$ & 0,5 & 0 & 1 & 0 & 1 \\
\hline Großstadtschule & $\begin{array}{l}\text { Schulen, die sich in Städten mit mehr als } 100.000 \text { Einwohnern } \\
\text { befinden, wurden mit dem Wert } 1 \text { kodiert. }\end{array}$ & $21,7 \%$ & 0,4 & 0 & 1 & 0 & 1 \\
\hline $\begin{array}{l}\text { mittlere Expositions- } \\
\text { zeit }\end{array}$ & $\begin{array}{l}\text { Die Angaben von Schülerinnen und Schülern zur Expositions- } \\
\text { zeit, die derselben Schule angehören, wurden aggregiert. }\end{array}$ & 30,8 & 2,2 & 26,7 & 35,8 & 29,1 & 32,4 \\
\hline $\begin{array}{l}\text { mittlere sportliche } \\
\text { Aktivität }\end{array}$ & $\begin{array}{l}\text { Die Angaben von Schülerinnen und Schülern zur sportlichen } \\
\text { Aktivität, die derselben Schule angehören, wurden aggre- } \\
\text { giert. }\end{array}$ & 6,7 & 1,2 & 3 & 10 & 5,9 & 7,5 \\
\hline
\end{tabular}


- Tab. 1 Fortzetzung

\begin{tabular}{|c|c|c|c|c|c|c|c|}
\hline \multicolumn{8}{|c|}{ Unabhängige Variablen (Kontextmerkmale, Fortsetzung) } \\
\hline Merkmal & Konstruktion & $\bar{x} / p$ & sd & $\min$ & $\max$ & $p(25)$ & $p(75)$ \\
\hline $\begin{array}{l}\text { mittlere Zustandsbe- } \\
\text { wertung }\end{array}$ & $\begin{array}{l}\text { F: Und wenn Du an Deine Schule denkst, wie sehr stimmen da } \\
\text { die folgenden Aussagen? Unsere Schule ist insgesamt in } \\
\text { einem guten Zustand A: stimmt überhaupt nicht zu; stimmt } \\
\text { eher nicht zu; teils/teils; stimmt ziemlich; stimmt genau } \\
\text { Die Antworten wurden auf einen Wertebereich von } 0 \text { (stimmt } \\
\text { überhaupt nicht) bis } 4 \text { (stimmt genau) rekodiert. Anschlie- } \\
\text { ßend wurden die Angaben von Schülerinnen und Schülern } \\
\text { zum schulischen Zustand, die derselben Schule angehören, } \\
\text { aggregiert. }\end{array}$ & 3,0 & 0,3 & 1,9 & 3,8 & 2,8 & 3,2 \\
\hline $\begin{array}{l}\text { Anteil an Kindern mit } \\
\text { häufiger Mobbinger- } \\
\text { fahrung }\end{array}$ & $\begin{array}{l}\text { Diese Variable repräsentiert den Anteil an Schülerinnen und } \\
\text { Schülern derselben Schule, die angegeben haben, 2- bis 3-mal } \\
\text { im Monat oder häufiger gemobbt worden zu sein. }\end{array}$ & $13,3 \%$ & 0,1 & $0,0 \%$ & $44,4 \%$ & $7,4 \%$ & $16,7 \%$ \\
\hline Anteil an Jungen & $\begin{array}{l}\text { Diese Variable repräsentiert den Anteil an männlichen } \\
\text { Schulkindern in den teilnehmenden Schulen. }\end{array}$ & $47,2 \%$ & 0,1 & $0,0 \%$ & $100 \%$ & $41,7 \%$ & $53,1 \%$ \\
\hline $\begin{array}{l}\text { Anteil an Kindern mit } \\
\text { Migrations-hinter- } \\
\text { grund }\end{array}$ & $\begin{array}{l}\text { Diese Variable repräsentiert den Anteil an Schülerinnen und } \\
\text { Schülern mit Migrationshintergrund in den teilnehmenden } \\
\text { Schulen. }\end{array}$ & $29,1 \%$ & 0,2 & $0,0 \%$ & $84,6 \%$ & $15,4 \%$ & $38,7 \%$ \\
\hline
\end{tabular}

Bei der Expositionszeit und der sportlichen Aktivität sind solche Effekte der Kontextvariablen jedoch deutlich zu erkennen. Dabei absorbiert die Expositionszeit auf Kontextebene den in Modell 2 deutlich stärker ausgeprägten Individualeffekt weitgehend. Gegenläufige Effekte zeigen sich bei der sportlichen Aktivität in Freizeit und Verein: Erhöht eine stärkere individuelle sportliche Betätigung die Wahrscheinlichkeit, von Verletzungen auf dem Schulhof betroffen zu sein, so ist der Effekt der Kontextvariable negativ. Die naheliegende Interpretation, wonach sich die individuelle sportliche Aktivität nur dann in einer höheren Verletzungsgefahr niederschlägt, wenn im Umfeld nur wenig Sport getrieben wird, scheidet jedoch aus, da eine sogenannte Cross-Level-Interaktion beider Sportvariablen nicht existiert. ${ }^{8}$ Somit wirken beide Variablen unabhängig voneinander.

Schließlich erweist sich die mittlere Bewertung des schulischen Zustands durch die Schüler/innen derselben Schule als ein signifikanter Prädiktor des Verletzungsgeschehens auf dem Schulhof. Schulkinder, die an Schulen unterrichtet werden, deren Zustand im Mittel besser eingeschätzt wird, weisen demnach eine geringere Chance auf, Verletzungen auf dem Schulhof zu erleiden.

Nach der Integration der Kontextmerkmale hat sich die verbleibende Varianz auf der Ebene der Schule nochmals deutlich verringert und fällt nunmehr so gering aus, dass sie nicht mehr signifikant von null verschieden ist. Zugleich ist Modell 3 dem Modell, das ausschließlich Individualmerkmale enthielt, hinsichtlich der Anpassung an die vorliegenden Daten signifikant überlegen. Ferner spricht für die Güte des vollständigen Modells 3, dass auf seiner Grundlage Wahrscheinlichkeiten für Verletzungen auf dem Schul-

\footnotetext{
${ }^{8}$ Dies wurde lediglich geprüft und ist im Modell nicht ausgewiesen. Allerdings zeigt sich, dass die Homogenität bzw. Heterogenität der Schülerschaft mit Blick auf sportliche Aktivität einen Einfluss auf die Prävalenz von Verletzungen auf dem Schulhof nimmt. So weist die (schulspezifische) Streuung der sportlichen Aktivität einen positiven und statistisch signifikanten Effekt auf die abhängige Variable aus (ebenfalls nicht im Modell ausgewiesen). Je heterogener die Schülerschaft im Hinblick auf die sportliche Aktivität ausfällt, desto höher ist demnach die Wahrscheinlichkeit von Verletzungen auf dem Schulhof.
}

hof kalkuliert werden, die sich zwischen einem und $42 \%$ bewegen. Auf der anderen Seite fällt die Streuung dieser geschätzten Wahrscheinlichkeiten im Schwerpunkt der Daten mit 3,2 (25. Perzentil) und $7,1 \%$ (75. Perzentil) nicht besonders hoch aus. Dies deutet darauf hin, dass es noch andere gewichtige Faktoren gibt, die für die Erklärung von Verletzungen auf dem Schulhof verantwortlich sind.

\section{Diskussion}

Auf Basis der vorliegenden Modelle konnten einige einflussreiche Risikofaktoren für Verletzungen auf dem Schulhof identifiziert werden. So weisen Jungen ein höheres Verletzungsrisiko auf als Mädchen. Zudem verletzen sich besonders Kinder, die häufig gemobbt werden, sportlich aktiv sind und/oder unter Schlafstörungen leiden häufiger.

Betrachtet man die Ergebnisse anderer Studien, so zeigt sich ein uneinheitliches Bild. Während Mattila et al. [3] ebenfalls ein erhöhtes (allgemeines) Verletzungsrisiko bei Kindern mit höherer sportlicher Aktivität berichten, spielt sportliche Betätigung bei Gofin et al. [5] keine Rolle für die Prävalenz von Schulunfällen. In einem nationalen Forschungsprojekt [12] zeigt sich gar ein selteneres Auftreten von Schulunfällen unter sportlich aktiven Kindern. Birken und MacArthur [18] berichten Effekte des sozioökonomischen Status und Kahl et al. [19] weisen einen Einfluss des Migrationshintergrunds auf das Verletzungsgeschehen nach. In der vorliegenden Untersuchung zeigen sich jedoch keine Effekte dieser beiden Merkmale.

In Bezug auf die übrigen Individualmerkmale ist die Befundlage hingegen eindeutiger: Demnach verletzen sich Jungen häufiger in der Schule als Mädchen [12-16]. Chau [14] bestätigt zudem, dass Schlafstörungen die Gefahr allgemeiner Verletzungen erhöhen während Beck et al. [17] zu dem Schluss kommen, dass Kinder mit Mobbingerfahrungen häufiger allgemeine Verletzungen erleiden.

Auf Kontextebene ist neben der Expositionszeit v. a. der schulische Zustand für das Verletzungsaufkommen auf dem Schulhof relevant. Regionale Unterschiede oder Effekte der Schulform konnten hingegen nicht nachgewiesen werden. Mit Blick auf die Schul- 


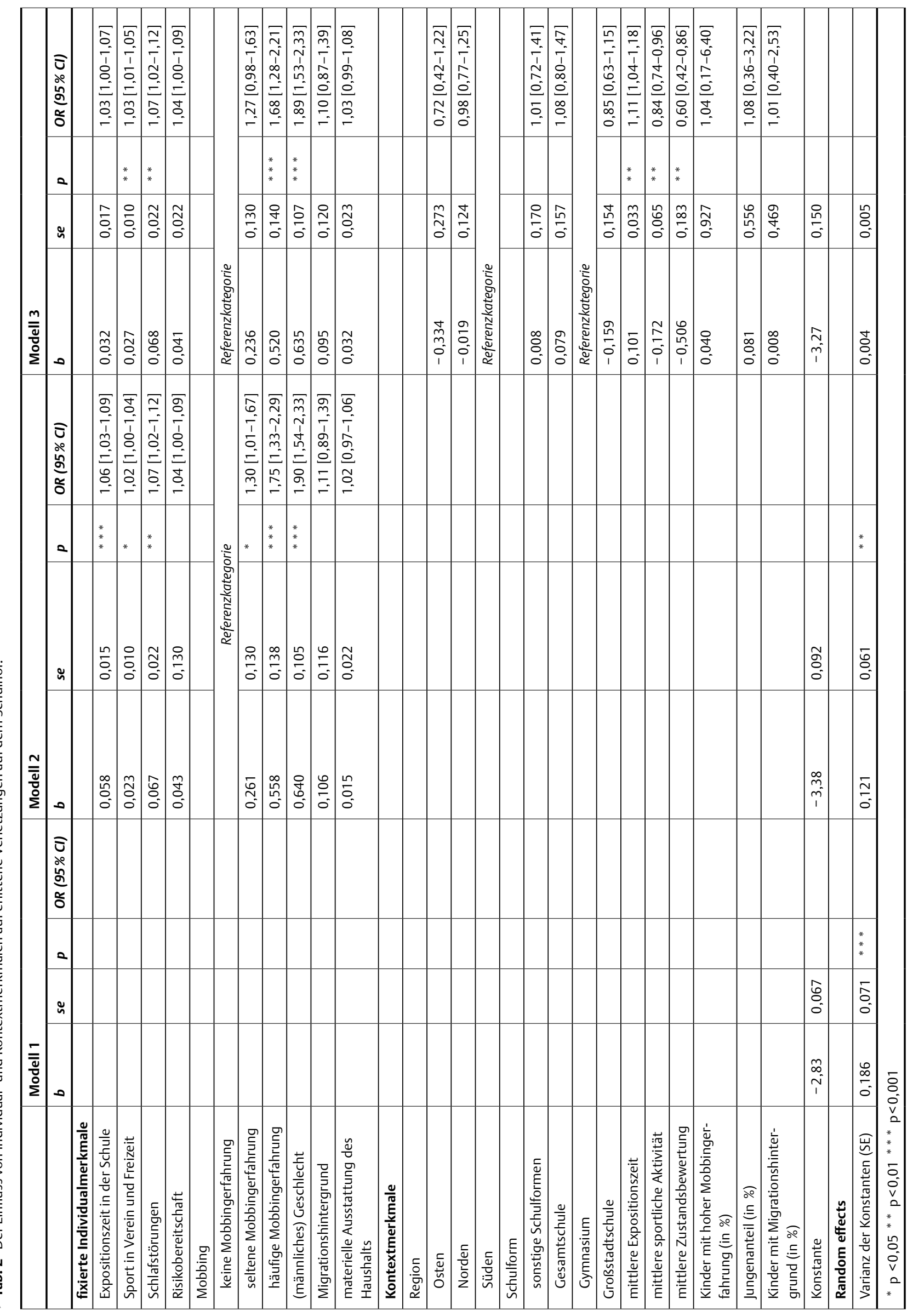


form deckt sich dieser Befund mit Ergebnissen aus anderen Studien $[12,20]$, während regionale Unterschiede bei Verletzungen im Kindesalter an anderer Stelle sehr wohl vorzufinden sind [3,22].

Neben der Darlegung konkreter Wirkungszusammenhänge möchte der vorliegende Beitrag auch dafür sensibilisieren, die verschiedenen Verletzungsorte im Kontext Schule differenziert zu betrachten. ${ }^{9}$ Mädchen verletzen sich bspw. häufiger im Schulsport, während Jungen häufiger Verletzungen auf dem Schulhof erleiden (vgl. auch [12, 21]). Obgleich der Schulhof beim Verletzungsaufkommen eine große Rolle spielt, wurde er bisher (im Gegensatz etwa zum Schulsport [23]-[24]) nur unzureichend in den Blick genommen. Hier bessert der vorliegende Beitrag nach.

Einschränkend muss erwähnt werden, dass die vorliegenden Modelle nur begrenzt in der Lage sind, das Verletzungsgeschehen auf dem Schulhof abschließend zu erklären. Möglicherweise gibt es noch weitere Erklärungsvariablen, die hier nicht berücksichtigt werden konnten. Entsprechend ist für die kommenden Erhebungswellen vorgesehen, das Verhalten der Schüler/innen während der Pause konkreter abzufragen. Da Verletzungen in vielen Fällen aber auch das Ergebnis von Pech und Zufall sein dürften, erscheint die insgesamt zu erklärende Variation jedoch generell begrenzt zu sein.

Limitationen der Studie ergeben sich auch aus dem Umstand, dass es sich mit Blick auf Verletzungen um selbstberichtete Daten mit einem langen Rückerinnerungszeitraum von 12 Monaten handelt. Dies legt eine gewisse Unschärfe der Angaben nahe. Des Weiteren umfasst die Stichprobe lediglich 138 verschiedene Schulen, was eine Einschränkung der Möglichkeiten bedeutet, Kontexteffekte erschöpfend zu modellieren.

Nichtsdestotrotz macht die Untersuchung deutlich, dass wirksame Präventionsstrategien von Verletzungen sowohl individuelle als auch kontextuelle Faktoren berücksichtigen müssen, so z. B. Mobbingstrukturen in der Schule oder den schulischen Zustand. Womöglich ergeben sich weitere wertvolle Hinweisen, wenn in den kommenden Jahren das volle Potenzial des Panelcharakters der Studie ausgeschöpft werden kann.

\section{Interessenkonflikt}

Die Autoren geben an, dass kein Interessenkonflikt besteht.

\section{Literatur}

[1] Ellsäßer G. Unfälle, Gewalt, Selbstverletzung bei Kindern und Jugendlichen 2014. Ergebnisse der amtlichen Statistik zum Unfallgeschehen 2012. Fachbericht. Wiesbaden: Statistisches Bundesamt; 2014

[2] Deutsche Gesetzliche Unfallversicherung (DGUV). Statistik Schülerunfallgeschehen 2014. 2016; Im Internet: http://www.dguv.de/medien/ inhalt/zahlen/documents/schueler/statistik_info_2014.pdf Stand: 17.02.2017

[3] Mattila V, Parkkari ], Kannus P et al. Occurrence and risk factors of unintentional injuries among 12- to 18-year old Finns - A survey of 8219 adolescents. European Journal of Epidemiology 2004; 19: 437-444

\footnotetext{
${ }^{9}$ Die Ergebnisse für andere Verletzungsorte sind hier nicht ausgewiesen.
}

[4] Pickett W, Garner M], Boyce WF et al. Gradients in risk for youth injury associated with multiple-risk behaviours: a study of 11,329 Canadian adolescents. Social Science \& Medicine 2002; 55: 1055-1068

[5] Gofin R, Donchin M, Schulrof B. Motor ability: protective or risk for school injuries? Accident Analysis and Prevention 2004; 36: 43-48

[6] Bergström E, Björnstig U. School Injuries. Epidemiology and clinical features of 307 cases registered at hospital during one school year. Scandinavian Journal of Primary Health Care 1991; 9: 209-216

[7] Chau N, Predine R, Benamghar L et al. Determinants of school injury proneness in adolescents: a prospective study. Public Health 2008; 122: $801-808$

[8] Sosnowska S, Kostka T. Epidemiology of school accidents during a six school-year period in one region in Poland. European Journal of Epidemiology 2003; 18: 977-982

[9] Gratz RR. School injuries: What we know, what we need. Journal of Pediatric Health Care 1992; 6: 256-262

[10] Hox ]. Multilevel analysis: techniques and applications. Mahwah, London: Erlbaum; 2002

[11] Rabe-Hesketh S, Skrondal A. Multilevel and longitudinal modeling using stata. Volume II: Categorical responses, Counts, and Survival. 3. Aufl College Station: Stata Press; 2012

[12] Universität Konstanz, Karlsruher Institut für Technologie, Unfallkasse Baden-Württemberg. Allgemeines Unfallgeschehen, Unfallgeschehen in der Schule und Aktivitätsverhalten bei Kindern und Jugendlichen. Abschlussbericht des Projekts Sportliche Aktivität, Fitness, Umwelt/ Mobilität und Unfälle bei Kindern und Jugendlichen. 2013; Im Internet: http://www.ukbw.de/sicherheit-gesundheit/betriebsarten/schulen/ Stand 17.02.2017

[13] Sun Y-H, Su IT-S, Wong T-W et al. Unintentional injuries at school in China - Patterns and risk factors. Accident Analysis and Prevention 2006; 38: 208-214

[14] Chau K. Impact of sleep difficulty on single and repeated injuries in adolescents. Accident Analysis and Prevention 2015; 81: 86-95

[15] Pagano A, Cabrini E, Anelli M et al. Accidents in the school environment in milan, a five year survey. European Journal of Epidemiology 1987; 3: 196-201

[16] Kraus R, Horas U, Szalay G et al. School-related injuries: a retrospective 5-year evaluation. European Journal of Trauma and Emergency Surgery 2011; 37: 411-418

[17] Beck NI, Arif I, Paumier MF et al. Adolescent injuries in Argentina, Bolivia, Chile, and Uruguay: Results from the 2012-2013 Global School-based Student Health Survey (GSHS). Injury 2016; 47: 2642-2649

[18] Birken CS, MacArthur C. Socioeconomic status and injury risk in children. Paediatrics and Child Health 2004; 9: 323-325

[19] Kahl H, Dortschy R, Ellsäßer G. Verletzungen bei Kindern und Jugendlichen (1-17 Jahre) und Umsetzung von persönlichen Schutzmaßnahmen. Bundesgesundheitsblatt- Bundesgesundheitsforschung-Gesundheitsschutz 2007; 50: 718-727

[20] Salminen S, Kurenniemi M, Råback M et al. School environment and school injuries. Frontiers in Public Health 2014; 1: 1-5

[21] Salminen S, Lounamaa A, Kurenniemi M. Gender and injury in Finnish comprehensive schools. Accident Analysis and Prevention 2008; 40: 1267-1272

[22] Dieterich S, Hofmann R, Hübner H. Schulunfälle im regionalen Vergleich. Public Health Forum 2016; 24: 33-35

[23] Dieterich S, Hense I, Hübner $\mathrm{H}$ et al. Das schulsportliche Unfallgeschehen in Nordrhein-Westfalen im Schuljahr 2008/09: Ergebnisse und Trends. 1. Aufl. Berlin. Münster: Lit; 2010.

[24] Hübner H, Pfitzner M. Sportverletzungen bei Kindern und Jugendlichen: Das schulsportliche Unfallgeschehen in Deutschland. Sports Orthopaedics and Traumatology Sport-Orthopädie - Sport-Traumatologie 2005; 21: 263-268 